\title{
Analysis of multistatic vehicle-drone Ground Penetrating Radar configurations for mine detection
}

\author{
Maria Garcia-Fernandez, Yuri Alvarez-Lopez, Fernando Las Heras \\ Area of Signal Theory and Communications \\ University of Oviedo \\ Gijón, Asturias, Spain \\ garciafmaria@uniovi.es
}

\author{
Ann Morgenthaler, Carey Rappaport \\ ALERT Center of Excellence \\ Northeastern University \\ Boston, Massachusetts, USA \\ c.rappaport@northeastern.edu
}

\begin{abstract}
In this contribution, a novel Ground Penetrating Radar (GPR) configuration to detect low-contrast buried targets is presented. It is based on combining the advantages of ForwardLooking GPR (FLGPR) and Down-Looking GPR (DLGPR) using a distributed GPR system, with a vehicle-mounted transmitter looking ahead and a drone-mounted receiver looking downwards. This configuration provides good penetration and high resolution, reducing the clutter compared to DLGPR. Several simulations have been performed and the resulting Synthetic Aperture Radar (SAR) images have been compared.
\end{abstract}

\section{INTRODUCTION}

Ground Penetrating Radar (GPR) is a well-known technique for subsurface imaging applications, such as pavement inspection, archaeological survey, or landmines and Improvised Explosive Devices (IEDs) detection [1]. The main advantages of GPR over other non-invasive techniques are that it can detect both metallic and non-metallic targets, and it can provide radar images of the underground. However, its performance is greatly affected by clutter mainly due to the strong reflections from the rough soil surface.

For the targeted application, the GPR system must keep a safety distance to avoid the accidental detonation of mines and IEDs. The typical approach to overcome this issue consists of a vehicle-mounted Forward-Looking GPR (FLGPR) [2]. In this configuration the antennas look ahead of the vehicle, almost parallel to the soil surface. This helps to mitigate the clutter since the specular reflection from the soil surface is in the opposite direction of the antennas. However, main drawbacks are the low sensitivity and the low height resolution (challenging the distinction between targets above or below the surface). Recently, another approach based on a drone-mounted Downward-Looking GPR (DLGPR) has been proposed [3]. In DLGPR the antennas are orthogonal to the soil surface, providing higher height resolution but suffering from stronger clutter (due to the specular reflection from the soil surface backscattered to the radar).

In order to reduce the clutter and improve the resolution, a novel GPR configuration that exploits the advantages of both FLGPR and DLGPR is proposed. It consists of a distributed GPR system, with the transmitter and the receiver antennas separated several meters. The transmitter looks ahead of the vehicle (resembling FLGPR systems), whereas the receiver looks downwards (following a DLGPR configuration). This article is devoted to analyzing the performance of this configuration for detecting plastic IEDs. The scattered field from a dielectric object buried under a rough ground surface has been simulated using Finite-Difference Frequency-Domain (FDFD) method [4]. Then, it has been post-processed with a Synthetic Aperture Radar (SAR) algorithm, and the resulting radar images have been compared.

\section{Methodology}

\section{A. Scattered field simulation}

The FDFD method has been used to simulate the 2D GPR scenario shown in Fig. 1. A multistatic configuration has been considered: the transmitter (TX) is placed at position $\boldsymbol{r}_{t}$ (with an angle of incidence $\theta_{i n c}$ ), and the receiver (RX) is looking downwards and is moved between $R$ positions $\boldsymbol{r}_{r}$ $(r=1, \ldots, R)$. The soil is characterized by its constitutive parameters $\left(\varepsilon_{r_{s}}\right.$ and $\left.\sigma_{s}\right)$ and its roughness (average height $h_{s}$ and correlation length $L_{s}$ ). The target has a circular shape with radius $\delta_{t g}$ and is centered at $\left(x_{t g}, y_{t g}\right)$. It is also characterized by its constitutive parameters $\left(\varepsilon_{r_{t g}}\right.$ and $\left.\sigma_{t g}\right)$. Simulations have been performed for both TE (Transverse Electric) and TM (Transverse Magnetic) polarization at $N$ frequencies. It must be noticed that in the FDFD simulations the contribution of the flat surface to the scattered field is removed.

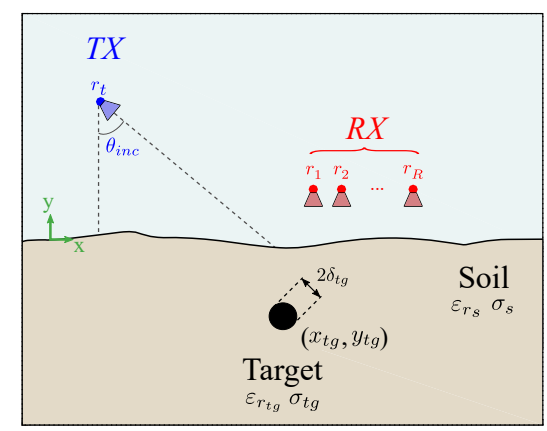

Fig. 1. Simulated 2D multistatic GPR scenario

\section{B. Inversion method}

As aforementioned, the simulated scattered field is postprocessed with a SAR algorithm. The SAR image value at 


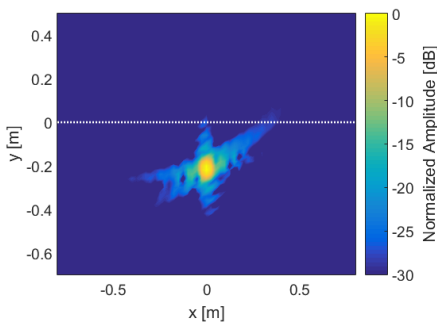

(a) $\mathrm{TE}$

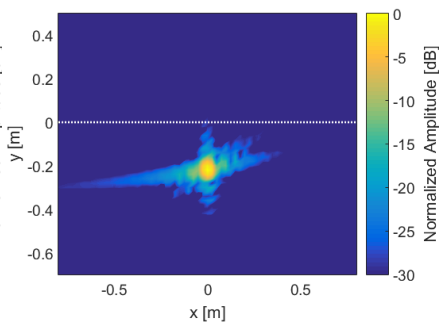

(b) $\mathrm{TM}$
Fig. 2. SAR image with low grazing incidence considering a flat surface.

point $r^{\prime}$ is given by 11 , where $R_{r}$ denotes the path length from the transmitter (at $\boldsymbol{r}_{t}$ ) to the $r$-th receiver (at $\boldsymbol{r}_{r}$ ) and passing through the point $\boldsymbol{r}^{\prime}$.

$$
\rho\left(\boldsymbol{r}^{\prime}\right)=\sum_{n=1}^{N} \sum_{r=1}^{R} E_{\text {scatt }}\left(f_{n}, \boldsymbol{r}_{r}^{t}\right) \exp \left(j \beta_{0} R_{r}\right)
$$

In order to obtain a well-focused SAR image with the buried target detected at its real depth, $R_{r}$ is calculated taking into account the dielectric permittivity of the soil.

\section{RESUlts}

In this contribution, the scenario is composed by a $2-\mathrm{cm}$ radius dielectric target (made of TNT with $\epsilon_{r_{t g}}=2.9$ and $\left.\sigma_{t g}=0 \mathrm{~S} / \mathrm{m}\right)$ buried at $20 \mathrm{~cm}$ depth in a sandy soil $\left(\varepsilon_{r_{s}}=2.5\right.$ and $\sigma_{s}=0.0125 \mathrm{~S} / \mathrm{m}$ ). The target is centered on the x-axis (thus, $x_{t g}=0 \mathrm{~m}$ and $y_{t g}=-0.20 \mathrm{~m}$ ). The scattered field is simulated at 201 frequencies between 3.5 and $5.5 \mathrm{GHz}$. It must be noticed that due to the low contrast between the soil and the target, it is practically undetectable using common FLGPR or DLGPR configurations.

\section{A. Low grazing incidence}

First, the TX has been oriented with an angle of incidence of $83^{\circ}$ (close to grazing incidence such as in FLGPR systems). It has been placed at $x=-20 \mathrm{~m}$ and $y=2.5 \mathrm{~m}$, whereas the receiver has been placed at $1 \mathrm{~m}$ height and moved between $x=-0.8$ and $x=0.8 \mathrm{~m}$. This geometry helps to properly illuminate the target.

The resulting SAR images are shown in Fig. 2 for both TE and TM polarizations considering a flat soil surface (depicted with a dotted white line). The maximum of the SAR image power is $\approx 15 \mathrm{~dB}$ higher for TM than for TE, making easier the detection of the target.

Fig. 3 shows the SAR images when the target is buried under a rough surface $\left(h_{s}=2 \mathrm{~cm}\right.$ and $\left.L_{s}=80 \mathrm{~cm}\right)$. In this case, the maximum is $\approx 7 \mathrm{~dB}$ higher for TM. Both the clutter and the target have higher reflectivity for TM, so the resulting signal to clutter ratio is almost the same as for TE. This might be affected by the fact that the simulation is $2 \mathrm{D}$.

\section{B. Brewster's angle incidence}

Then, another geometry with an angle of incidence closer to Brewster angle $\left(\approx 58^{\circ}\right)$ has been analyzed. For this angle

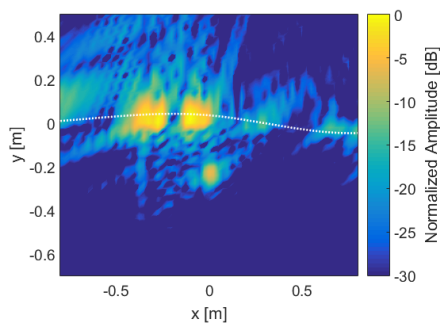

(a) $\mathrm{TE}$

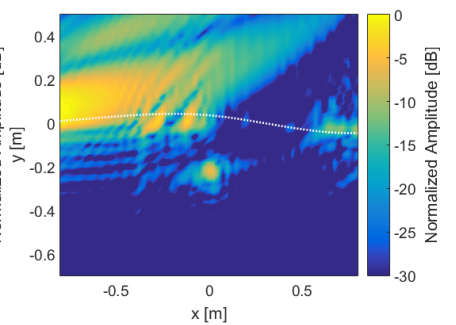

(b) TM
Fig. 3. SAR image with low grazing incidence considering a rough surface.

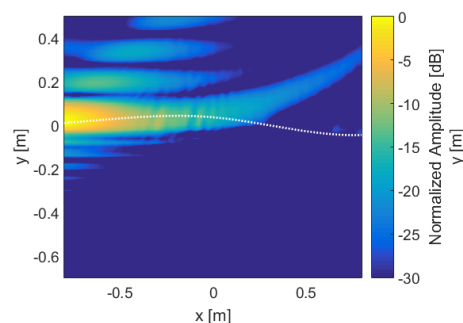

(a) $\mathrm{TE}$

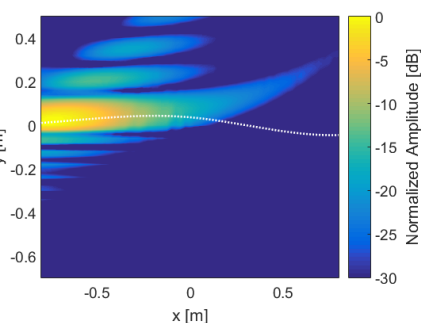

(b) TM
Fig. 4. SAR image for Brewster's angle considering a rough surface.

of incidence, TM waves are ideally perfectly transmitted into the soil (without reflection). The TX and RX are placed closer (with the TX at $x=-4 \mathrm{~m}$ ) to properly illuminate the target.

SAR images are depicted in Fig. 4 considering the same rough surface. The target cannot be detected even for TM polarization. This might be due to the lower angle of incidence and the closer distance between TX-RX, which could imply that more rays reflected by rough surface points are received.

\section{CONCLUSION}

A novel GPR configuration has been analyzed to detect a TNT target buried in sandy soil. Simulations show that orienting the transmitter with a low grazing incidence helps to reduce the clutter backscattered to the receiver, thus enabling the detection of the target in the SAR image.

\section{ACKNOWLEDGEMENT}

This work has been partially supported by Government of Spain (project TEC2014-55290-JIN, and grants FPU15/06341 and EST17/0777) and Government of Asturias (project GRUPIN-18-000191).

\section{REFERENCES}

[1] D. J. Daniels, Ground Penetrating Radar, 2nd ed. London: IET, 2004.

[2] D. Comite, F. Ahmad, M. G. Amin, T. Dogaru, "Detection of lowsignature targets in rough surface terrain for forward-looking ground penetrating radar imaging", in 49th Asilomar Conference on Signals, Systems and Computers, 2015.

[3] M. Garcia-Fernandez, Y. Alvarez-Lopez, A. Arboleya-Arboleya, B. Gonzalez-Valdes, Y. Rodriguez-Vaqueiro, F. Las Heras, A. Pino, "Synthetic Aperture Radar imaging system for landmine detection using a Ground Penetrating Radar on board an Unmanned Aerial Vehicle", IEEE Access, vol. 6, pp. 45100-45112, 2018.

[4] C. Rappaport, Q. Dong, E. Bishop, A. Morgenthaler, M. Kilmer, "Finite Difference Frequency Domain (FDFD) Modeling of Two Dimensional TE Wave Propagation and Scattering," in 2004 URSI Conference, 2004. 